\title{
Medium-Long-Chain Chimeric Human Acyl-CoA Dehydrogenase: Medium-Chain Enzyme with the Active Center Base Arrangement of Long-Chain Acyl-CoA Dehydrogenase $^{\dagger}$
}

\author{
Andreas Nandy ${ }^{\ddagger}$ Volker Kieweg, ${ }^{\ddagger}$ Franz-Georg Kräutle, ${ }^{\ddagger}$ Petra Vock,, Burkhard Küchler,, Peter Bross, ${ }^{\S}$ \\ Jung-Ja P. Kim," Ihab Rasched, \\ Faculty of Biology, University of Konstanz, P.O. Box 5560-M644, D-78434 Konstanz, Germany, Department of Clinical \\ Chemistry, Molecular Genetic Laboratory, Skejby Sygehus, DK-8200 Århus N, Denmark, and Department of Biochemistry, \\ Medical College of Wisconsin, Milwaukee, Wisconsin 53226
}

\begin{abstract}
The catalytically essential glutamate residue that initiates catalysis by abstracting the substrate $\alpha$-hydrogen as $\mathrm{H}^{+}$is located at position 376 (mature MCADH numbering) on loop JK in medium chain acyl-CoA dehydrogenase (MCADH). In long chain acyl-CoA dehydrogenase (LCADH) and isovalerylCoA dehydrogenase (IVDH), the corresponding Glu carrying out the same function is placed at position 255 on the adjacent helix G. These glutamates thus act on substrate approaching from two opposite regions at the active center. We have implemented the topology of LCADH in MCADH by carrying out the two mutations Glu376Gly and Thr255Glu. The resulting chimeric enzyme, "medium-/long" chain acyl-CoA dehydrogenase (MLCADH) has $\sim 20 \%$ of the activity of MCADH and $\sim 25 \%$ that of LCADH with its best substrates octanoyl-CoA and dodecanoyl-CoA, respectively. MLCADH exhibits an enhanced rate of reoxidation with oxygen, however, with a much narrower substrate chain length specificity that peaks with dodecanoyl-CoA. This is the same maximum as that of LCADH and is thus significantly shifted from that of native MCADH (hexanoyl/octanoyl-CoA). The putative, common ancestor of LCADH and IVDH has two Glu residues, one each at positions 255 and 376. The corresponding MCADH mutant, Thr255Glu (glu/glu-MCADH), is as active as MCADH with octanoyl-CoA; its activity/chain length profile is, however, much narrower. The topology of the Glu as $\mathrm{H}^{+}$abstracting base seems an important factor in determining chain length specificity and reactivity in acyl-CoA dehydrogenases. The mechanisms underlying these effects are discussed in view of the three-dimensional structure of MLCADH, which is presented in the accompanying paper [Lee et al. (1996) Biochemistry 35, 12412-12420].
\end{abstract}

Acyl-CoA dehydrogenases are a class of flavoproteins that catalyze the desaturation of acyl-CoA substrates. Four known members are involved in mammalian mitochondrial $\beta$-oxidation of fatty acids (short-, medium-, long-, and very long chain acyl-CoA dehydrogenases), and three (isovaleryl-, isobutyryl- and glutaryl-CoA dehydrogenases) are involved in the degradation of amino acids. In addition microsomal or peroxisomal acyl-CoA oxidases and a variety of related enzymes of bacterial or plant origin can be viewed as sharing the capacity to catalyze the seemingly identical chemical process, the substrate dehydrogenation at the positions $\alpha, \beta$. All these enzymes vary widely in their substrate specificity and in the nature of their electron acceptors and have accordingly received appropriate names. The best-studied member of the extended family is MCADH which has achieved prominence, since it has a very high incidence of genetic defects in humans (Gregersen, 1993). At the active center of MCADH there is a glutamic acid residue (Glu376)

\footnotetext{
$\dagger$ This work was supported by grants from the Deutsche Forschungsgemeinschaft (Gh 2/4-7, Gh 2/6-1) to S.G. and from the National Institutes of Health (GM29076) to J.-J.P.K. Presented in part at the 11th International Symposium on Flavins and Flavoproteins, Nagoya, Japan (1993) (Nandy et al., 1994).

* To whom correspondence should be addressed.

$¥$ University of Konstanz.

$\S$ Skejby Sygehus.

"Medical College of Wisconsin.

${ }^{\otimes}$ Abstract published in Advance ACS Abstracts, September 1, 1996.
}

(Fendrich \& Abeles, 1982; Powell \& Thorpe, 1988; Kim et al., 1993), which has been shown to initiate catalysis by abstracting the substrate $\alpha$-hydrogen as $\mathrm{H}^{+}$(Ghisla et al., 1984, 1994; Bross et al., 1990). ${ }^{1}$

The amino acid sequence is known for 39 proteins. There are 15 mammalian acyl-CoA dehydrogenases, one enoylCoA reductase (Ascaris suum), one butyryl-CoA dehydrogenase (Megasphaera elsdenii), one Caenorhabditis elegans dehydrogenase, four bacterial dehydrogenases, and 17 acylCoA oxidases (MIPS, 1995). A sequence alignment of the active site domains shows that the glutamate at the position corresponding to Glu376 of MCADH is conserved in all but seven sequences. The latter are those of three mammalian LCADH's, of 2 IVDH's, and of one Escherichia coli and one $C$. elegans sequences, where a glycine is found at the

\footnotetext{
${ }^{1}$ Abbreviations: CX-CoA, straight-chain acyl-CoA thioesters, where " $\mathrm{X}$ " denotes the length of the carbon chain; ADH, acyl-CoA dehydrogenase; IVDH, isovaleryl-CoA dehydrogenase; MCADH, human wild type medium-chain acyl-CoA dehydrogenase; MLCADH, human medium-long-chain acyl-CoA dehydrogenase $=$ T255E/E376G MCADH glu/glu-MCADH = T255E MCADH; LCADH, human wild type longchain acyl-CoA dehydrogenase; CT, charge transfer; $k_{\text {red }}, k_{\text {ox }}$, rate of enzyme flavin reduction and oxidation, respectively; $V_{\text {reox }}-\mathrm{O}_{2}$, velocity of enzyme reoxidation with dioxygen as the electron acceptor including all steps involved. Enzymes: MCADH, E. C. 1.3.99.3; LCADH, E.C. 1.3.99.13 MIPS. PIR Data Bank of the Martinsrieder Institut für Proteinsequenzen (MIPS), Martinsreid, Germany.
} 


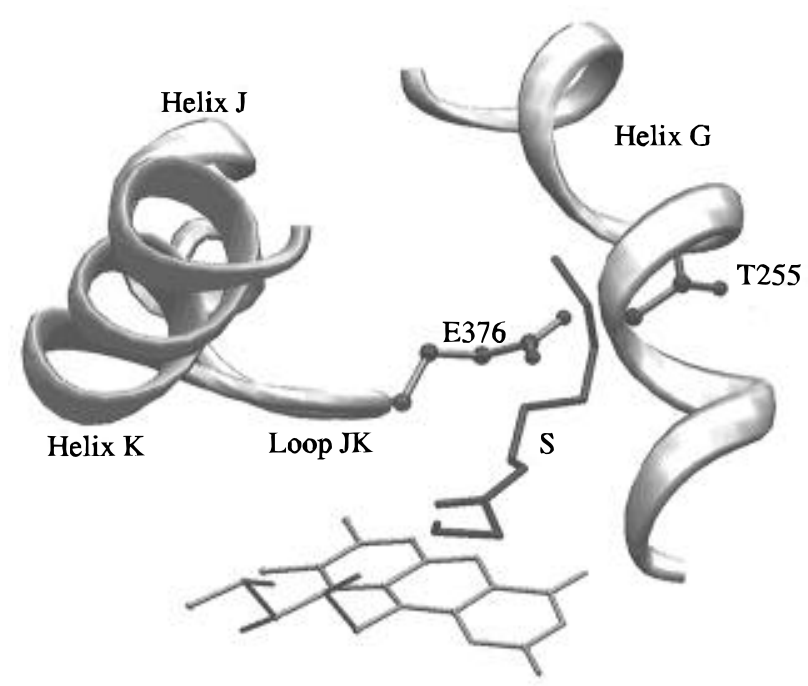

FIGURE 1: Three-dimensional representation of the arrangement of flavin cofactor, substrate, and $\mathrm{H}^{+}$-abstracting base at the active center of MCADH. The isoalloxazine moiety is at the bottom, and the octanoyl-COS- $\mathrm{CH}_{2}$ - portion of the substrate $(\mathrm{S})$ is placed above its plane. Glu376, the base initiating catalysis by abstraction of the substrate $\alpha-\mathrm{H}$ is linked to loop JK. Note that in the structure of LCADH a Glu would be located at the place of Thr 255 on helix G (Kim et al., 1994), from which position it can take on the function of Glu376. Note that in MLCADH the mutations Glu376Gly and Thr255Glu and in glu/glu-MCADH the mutation Thr255Glu have been implemented.

corresponding position (Nandy et al., 1996). It has thus been a puzzle as to which base in LCADH and IVDH plays the role of Glu376 (of MCADH) in the $\mathrm{H}^{+}$abstraction.

On the basis of the three-dimensional structure (Kim \& Wu, 1988), Kim et al. (1993) have proposed that the base in question is positioned on a different segment of the polypeptide chain, on the $\alpha$-helix G in LCADH, and that from this location the $\mathrm{Glu}_{-} \mathrm{COO}^{-}$can reach into a position functionally equivalent to that of Glu376, which lies on loop JK in MCADH (Figure 1). For LCADH this hypothesis has been verified by the results obtained with the Glu262Gln mutant (Djordjevic et al., 1994) (note that position 262 corresponds to position 255 in MCADH) . The LCADH numbering refers to the $\mathrm{NH}_{2}$-terminal sequence of mature pig LCADH, starting with SGGEERLE.

From this, several interconnected questions do arise:

(a) What is the reason for the different positions of the $\mathrm{H}^{+}$-abstracting base? Does it affect substrate chain length specificity, and are there any other active center functional groups participating in the latter?

(b) Is the "chemistry" underlying $\alpha, \beta$-dehydrogenation affected by the position of the base and how?

(c) From a phylogenetic point of view an ancestor enzyme could be postulated at a branching point in evolution, which carries Glu residues both at the positions 376 and 255 (Djordjevic et al., 1994; Nandy et al., 1996). Can this hypothesis be verified, and what are the properties of such an active site arrangement?

In order to provide a partial answer to these issues we have generated the following mutants of human MCADH in which the active site configuration of LCADH is implemented (in steps) in MCADH (Figure 1): glu/gluMCADH (mutation: T255E) the proposed common ancestor of LCADH and IVDH; T255E/E376T-MCADH in which a Thr and a Glu have interchanged positions; MLCADH in which the arrangement of functional groups at positions 255 and 376 is that present in LCADH, i.e., Thr255Glu/ Glu376Gly (Nandy et al., 1994). The properties of these proteins are compared to those of the parent MCADH and of LCADH. In the accompanying paper the 3D-structure of MLCADH is presented (Lee et al., 1996). Since our original report (Nandy et al., 1994), Mohsen and Vockley (1995) have provided a direct evidence for Glu254 being the catalytically essential base in IVDH. They have also implemented a double mutation leading to an IVDH with a Glu at a position similar to that in MCADH; in contrast to our case, however, this mutant appears to have low activity, and no clear-cut effects on the dependance of activity on the substrate chain length were observed.

\section{MATERIALS AND METHODS}

Materials and Enzymes. CoA derivatives were either from Sigma or were synthesized as indicated. 3-ThiaoctanoylCoA (Lau et al., 1988), 4-nitrophenyl-acetyl-CoA (Engst, 1993), 2-octynoyl-CoA (Powell \& Thorpe, 1988). Ferricenium hexafluorophosphate was prepared according to Lehman and Thorpe (1990). Restriction enzymes, Klenow enzyme, and ampicillin were from Boehringer Mannheim; mung bean nuclease was from USB; in vitro mutagenesis kit, E. coli TG1 cells, and pBlueScript plasmids were from Amersham; the plasmid pTrc99C was from Amann (Amann et al., 1988). The LCADH expression plasmid was a kind gift from Dr. A. W. Strauss (Washington University, of St. Louis, MO), the enzyme was expressed in Escherichia coli and purified essentially as described by Djordjevic et al. (1994).

Instrumentation and Conditions. Visible and UV spectra were recorded with a Kontron Uvikon 930 spectrophotometer. $\mathrm{NH}_{2}$-terminal amino acid sequencing was done with an Applied biosystems 477A instrument. Single-turnover experiments were performed with a stopped-flow spectrophotometer which has a $2.0 \mathrm{~cm}$ path length cell (Raichle, 1981; Langkau, 1993), a "dead time" of 3-4 ms, and is equipped with a diode array detector (Spectroscopy Instruments GmbH, D-82205 Gilching, Germany) and interfaced with a MacIntosh IIcx computer for data acquisition (POSMA 2.3k software from Spectroscopy Instruments). Rapid reactions were routinely recorded in the range $300-650 \mathrm{~nm}$ using the "normal scan mode" with a scan time of $10 \mathrm{~ms} /$ spectrum and with a resolution of 2 pixel $/ \mathrm{nm}$. For reactions proceeding with a $k_{\mathrm{obs}} \geq 100 \mathrm{~s}^{-1}$ a so-called "fast access" routine was used, which has an acquisition time of $0.7 \mathrm{~ms}$ at $450 \mathrm{~nm}$. For these experiments a normally used BG24A filter was removed to yield sufficient light intensity. From at least three reproducible kinetic traces obtained under the same conditions the arithmetical mean was formed and then fitted using program "A" (Dr. D. P. Ballou, University of Michigan, Ann Arbor, MI). Other fit-routines were carried out with "KaleidaGraph" (Synergy Software) and appropriate algorithms. Unless otherwise stated, all measurements were carried out in $100 \mathrm{mM} \mathrm{KP}$, pH 7.6, at $25{ }^{\circ} \mathrm{C}$ (standard buffer). The concentrations mentioned in the context of stopped flow experiments are those after mixing, i.e., 1/1 dilution.

Construction of the Expression Plasmid pTrc MCADH. The plasmid pWTMCAD-2 (Bross et al., 1990) was linearized with $N d e I$, the fragment ends were rendered blunt by fill-in with Klenow enzyme, and the linear plasmid was 
subsequently digested with HindIII. The fragment encompassing the MCADH sequence was gel purified. The pTrc99C vector plasmid (Amann et al., 1988), which contains an inducible trc promoter and the lacI repressor gene was digested with $\mathrm{NcoI}$ and subsequently treated with mung bean nuclease to generate blunt-ends; the linear vector was digested with HindIII and, after gel purification, ligated with the above fragment obtained from pWTMCAD-2. The resulting MCADH expression plasmid was designated $\mathrm{pTrc}$ MCADH.

Construction of the Active Site Mutants. The plasmid pGln-2 (Bross et al., 1990) was used to generate a series of mutations at position 376 via cassette mutagenesis exchanging the BglII/KpnI cassette with synthetic DNA having ambiguous nucleotides in the first two bases of the codon for amino acid 376 of MCADH. One of the products contained the E376G mutation. For the other point mutations, oligonucleotide-directed mutagenesis was performed using the in vitro mutagenesis system from Amersham. In brief, appropriate restriction fragments encoding different parts of wild type or E376G mutant MCADH were subcloned into pBlueScriptSKII(-) to allow production of single stranded templates for mutagenesis. These were utilized for the mutagenesis procedure using the oligonucleotides: 5'CTTTTGATAAAGAAAGACCTGTAGTAGC-3' (T255E) and 5'-CTATCAGATTTATACCGGTACTTCACAAATTC$3^{\prime}$ (E376T). After successful mutagenesis, restriction fragments containing the respective mutations were subcloned into the expression vector $\mathrm{pTrc} \mathrm{MCADH}$ replacing the wild type MCADH sequences and producing the following single and double mutant derivatives: pTrc MCADH T255E (glu/ glu-MCADH), pTrc MCADH T255E/E376G (MLCADH), and pTrc MCADH T255E/E376T.

MCADH Expression in E. coli. E. coli TG1 cells were transformed with the expression plasmid pTrc MCADH for wild type MCADH expression or with a mutant plasmid. Cells were grown in dYT medium containing $100 \mu \mathrm{g}$ of ampicillin/mL at a temperature of $28^{\circ} \mathrm{C}$ to avoid inclusion body formation. Expression of MCADH protein was induced at $\mathrm{OD}_{578}=1.0$ with $0.1 \mathrm{mM}$ isopropyl $\beta$-Dthiogalactopyranoside, and the E. coli cells were harvested after an induction time of about $16 \mathrm{~h}$ with an $\mathrm{OD}_{578}$ of 6-9.

Purification of Mutant MCADH's Expressed in E. coli, Handling, and "Degreening." The purification of MCADH and of active site mutants was carried out as will be described elsewhere, and the yield of mutants was essentially the same as that of MCADH. For storage, purified MCADH was incubated with a 5-fold molar excess of FAD for $30 \mathrm{~min}$ in buffer (50 mM Tris, pH 7.8, containing $200 \mathrm{mM} \mathrm{NaCl}$ ) on ice and brought to $85 \%$ ammonium sulfate, and the precipitate was stored at $4{ }^{\circ} \mathrm{C}$. Immediately prior to use, the enzyme was resuspended in the desired buffer and desalted over a Pharmacia Sephadex G-25 M column (bed volume $9.1 \mathrm{~mL}$ ). For "degreening" [removal of tightly bound CoA-persulfide (Williamson et al., 1982)] $200 \mu \mathrm{L}$ of a saturated solution of dithionite in the desired buffer was added to $800 \mu \mathrm{L}$ of a cold, concentrated MCADH suspension in the same buffer. The enzyme becomes colorless while it dissolves, and after 1-2 min it is passed through a Sephadex G-25 M column, during which process it reoxidizes to the yellow form.

Extinction Coefficients. Extinction coefficients of the oxidized enzyme flavin $\lambda_{\max }$ in the visible range were obtained using the SDS method, and these coefficients were used for determination of enzyme concentrations. To a solution of MCADH in $20 \mathrm{mM} \mathrm{NaP}$, $\mathrm{pH} 7.8$, SDS (final concentration $1 \%$ ) was added. The resulting spectrum of the flavin was recorded, and the $\epsilon_{\max }$ values were calculated based on $\epsilon_{450}=11.3 \mathrm{mM}^{-1} \mathrm{~cm}^{-1}$ for free FAD (Whitby, 1953). $\epsilon_{\max }: \mathrm{MCADH}=14.8 ; \mathrm{MLCADH}=13.8 ;$ glu/gluMCADH $=14.6$; and LCADH $=14.1 \mathrm{mM}^{-1} \mathrm{~cm}^{-1}$. Saturated acyl-CoA's: $\epsilon_{260}=15.4 \mathrm{mM}^{-1} \mathrm{~cm}^{-1}$ (Decker, 1959).

Enzyme Assay. MCADH activity was measured at $25^{\circ} \mathrm{C}$ with the ferricenium assay (Lehman \& Thorpe, 1990) in 100 $\mathrm{mM} \mathrm{KP}_{\mathrm{i}} \mathrm{pH} 7.6$ (standard buffer) using $200 \mu \mathrm{M}$ ferricenium, a concentration that was found to be saturating for all proteins tested.

Spectrophotometric Titrations and Determination of Dissociation Constants. These experiments were carried out in standard buffer at $25{ }^{\circ} \mathrm{C}$, unless indicated otherwise. Dissociation constants $K_{\mathrm{d}}$ were estimated from fits of plots of $\Delta \mathrm{OD} /[$ ligand], with necessary corrections due to [ligand] $\approx$ [ligand•enzyme] as described by Bisswanger (1994).

\section{RESULTS}

Construction of an Improved Expression System. We have previously used the plasmid pWTMCAD-2 for expression of human MCADH in E. coli. This plasmid produces relatively low amounts of active MCADH, making purification of the protein tedious. To obtain higher levels of expression of human MCADH in E. coli, a new expression vector was constructed as described in Materials and Methods. The new vector, pTrc MCADH, contains the MCADH cDNA fragment encoding the mature part of the protein under control of the trc promoter. Under appropriate growth conditions (see Materials and Methods) E. coli cells transformed with this plasmid yield high amounts of active MCADH ( 25 fold higher amounts than obtained earlier) (Bross et al., 1990). The correct size of the monomers was confirmed by SDS-PAGE (not shown) and the authenticity of the $\mathrm{NH}_{2}$-terminal end ( + methionine) was confirmed by sequencing.

Catalytic Properties and Substrate Chain Length Specificity. For the study of the catalytic activity, we have used the assay described by Lehman and Thorpe (1990), which utilizes ferricenium as the electron acceptor. The electron transfer properties of ferricenium are closest to those of electron-transferring flavoprotein, the physiological electron acceptor. The first salient result is that MLCADH and glu/ glu-MCADH are substantially active, with turnover rates of the same dimension as those of the parent MCADH (Figure 2 , Table 1). The second is the substantially altered dependence of the activity on the substrate chain length compared to that of MCADH. The activity of MCADH is maximal with C6- and C8-CoA (Figure 2A), while with MLCADH (Figure 2B) the maximum is shifted to C12- and C14-CoA and becomes close to that of LCADH. Furthermore, while the specificity of MCADH and of LCADH is broad, and stretches over six to eight $-\mathrm{CH}_{2}-\mathrm{CH}_{2}$ - units (Figure 2A), the specificity of both MLCADH and glu/glu-MCADH is much narrower and is limited to essentially two substrates (Figure 2B). Comparing glu/glu-MCADH with MCADH, the most striking difference is found for the activity with C6-CoA, suggesting a specific effect of the $-\mathrm{CH}_{2}-\mathrm{CH}_{2}$ - unit differentiating the two substrates. We have found this result 
Table 1: Kinetic Parameter of Wildtype and Mutant Human MCADH and of Wildtype Human LCADH in Dependence of the Substrate Chain Length $^{a}$

\begin{tabular}{|c|c|c|c|c|c|c|c|c|c|c|c|}
\hline \multirow{2}{*}{$\begin{array}{l}\text { enzyme and base } \\
\text { arrangement }\end{array}$} & \multirow{2}{*}{$\begin{array}{l}\text { parameter units } \\
\text { and conditions }{ }^{b}\end{array}$} & \multicolumn{10}{|c|}{ substrate (chain length) } \\
\hline & & $\mathrm{C} 4$ & C6 & $\mathrm{C} 8$ & $\mathrm{C} 10$ & $\mathrm{C} 12$ & $\mathrm{C} 14$ & $\mathrm{C} 16$ & $\mathrm{C} 18$ & $\mathrm{C} 20$ & iso-C5 \\
\hline \multirow[b]{2}{*}{ MCADH } & $V_{\max }\left[\min ^{-1}\right]$ & 500 & 1010 & 980 & 580 & 550 & 280 & 140 & $<10$ & $<2$ & $<1$ \\
\hline & $K_{\mathrm{m}}[\mu \mathrm{M}]$ & 175 & 15 & 3.4 & 2.5 & 2.5 & 2.3 & 1.6 & $f$ & $f$ & $f$ \\
\hline \multirow{5}{*}{55} & $V_{\max } / K_{\mathrm{m}}$ & 3 & 67 & 288 & 232 & 220 & 122 & 88 & $f$ & $f$ & $f$ \\
\hline & $\begin{array}{l}\% \text { red. }{ }^{c} \\
k_{\text {rd }}\left[\min ^{-1}\right]^{d}\end{array}$ & 86 & & 100 & 97 & 96 & & 58 & & & \\
\hline & $2^{\circ} \mathrm{C}$ & & & $\sim 24000$ & 15000 & 3000 & & & & & \\
\hline & $25^{\circ} \mathrm{C}$ & 360 & & $>20000$ & $>20000$ & 5400 & 420 & & & & \\
\hline & $V_{\text {reox }-\mathrm{O}_{2}}\left[\min ^{-1}\right]^{e}$ & 0.065 & 0.028 & 0.004 & 0.004 & 0.01 & 0.021 & 0.018 & & & \\
\hline \multirow{3}{*}{ glu/glu-MCADH } & $V_{\max }\left[\min ^{-1}\right]$ & 500 & 170 & 790 & 680 & $<10$ & $<10$ & $<5$ & $<5$ & $<5$ & 0 \\
\hline & $K_{\mathrm{m}}[\mu \mathrm{M}]$ & 70 & 1.4 & 8 & 10 & $f$ & $f$ & $f$ & $f$ & $f$ & \\
\hline & $V_{\max } / K_{\mathrm{m}}$ & 7 & 121 & 99 & 68 & $f$ & $f$ & $f$ & $f$ & $f$ & \\
\hline \multirow{3}{*}{$\begin{array}{c}255 \\
\mathrm{E} \mathrm{E}^{\prime}\end{array}$} & $\%$ red. ${ }^{c}$ & 87 & 95 & 100 & 86 & & & & & & \\
\hline & $25^{\circ} \mathrm{C}$ & & & $>20000$ & 1500 & $\approx 0$ & $\approx 0$ & & & & \\
\hline & $V_{\text {reox }-\mathrm{O}_{2}}\left[\min ^{-1}\right]^{e}$ & & & 0.005 & 0.002 & & & & & & \\
\hline \multirow{2}{*}{ T255E/E376T-MCADH } & $V_{\max }\left[\min ^{-1}\right]$ & 0 & 0 & $<5$ & 14 & 6 & $<5$ & $<5$ & $<2$ & $<2$ & 0 \\
\hline & $K_{\mathrm{m}}[\mu \mathrm{M}]$ & & & $f$ & 50 & 50 & $f$ & $f$ & $f$ & $f$ & \\
\hline \multirow{2}{*}{$\begin{array}{c}255 \\
E \\
T^{3}\end{array}$} & $V_{\max } / K_{\mathrm{m}}$ & & & $f$ & 0.3 & 0.1 & $f$ & $f$ & $f$ & $f$ & \\
\hline & $V_{\max }\left[\min ^{-1}\right]$ & 0 & $<5$ & 10 & 20 & 200 & 160 & 37 & $<5$ & $<5$ & 0 \\
\hline \multirow[t]{2}{*}{ MLCADH } & $K_{\mathrm{m}}[\mu \mathrm{M}]$ & & $f$ & 1.0 & 4.2 & 6.6 & 5.5 & 1.3 & $f$ & $f$ & \\
\hline & $V_{\max } / K_{\mathrm{m}}$ & & $f$ & 10 & 5 & 30 & 29 & 28 & $f$ & $f$ & \\
\hline \multirow{4}{*}{$\begin{array}{c}255 \\
\mathrm{E}^{\prime}\end{array}$} & $\%$ red. $^{c}$ & & 83 & 90 & 93 & 89 & 81 & 34 & 5 & & \\
\hline & $k_{\text {red }}\left[\min ^{-1}\right]^{d}$ & & & & & & & & & & \\
\hline & $25^{\circ} \mathrm{C}$ & & & 145 & 480 & 415 & 150 & & & & \\
\hline & $V_{\text {reox }-\mathrm{O}_{2}}\left[\mathrm{~min}^{-1}\right]^{e}$ & & 0.02 & 0.03 & 0.04 & 1.1 & 2.0 & 3.1 & & & \\
\hline LCADH & $V_{\max }\left[\min ^{-1}\right]^{g}$ & 0 & 380 & 450 & 700 & 700 & 400 & 360 & 320 & 45 & \\
\hline \multirow{2}{*}{$\begin{array}{c}262 \\
E G\end{array}$} & $K_{\mathrm{m}}[\mu \mathrm{M}]^{g}$ & - & 29 & 8 & 10 & 7 & 10 & 14 & 8 & & \\
\hline & $V_{\max } / K_{\mathrm{m}}{ }^{g}$ & - & 13 & 56 & 70 & 100 & 40 & 26 & 40 & & \\
\hline
\end{tabular}

${ }^{a}$ The cartoons represent the functional groups linked to helix $\mathrm{G}$ and/or loop JK and their spatial correlation to flavin and substrate. See also Figure $1 .{ }^{b}$ Turnover rates determined in standard buffer using the ferricenium assay (Lehman $\&$ Thorpe, 1990). $V_{\max }$ is expressed as molecules ferricenium reduced per FAD and minute. ${ }^{c} \%$ bleaching of the flavin absorbance at $450 \mathrm{~nm}$ observed upon addition of excess of substrate, after 2 min compared to the extent of bleaching of MCADH with excess octanoyl-CoA. In the latter case an $\epsilon_{450} \approx 2650 \mathrm{M}^{-1} \mathrm{~cm}^{-1}$ is observed, which corresponds to the absorbance of the charge transfer complex of fully reduced enzyme with the product octenoyl-CoA. Note that upon reduction of MCADH with dithionite a lower $\epsilon_{450} \approx 1500 \mathrm{M}^{-1} \mathrm{~cm}^{-1}$ is obtained. ${ }^{d}$ Rates of the first, major phase of a biphasic exponential absorption decrease at $450 \mathrm{~nm}$ obtained from "stopped flow" measurements. ${ }^{e}$ Increase of absorption at $450 \mathrm{~nm}$ following aerobic reduction with $\sim 0.7$ equiv of substrate ( $c f$. also legend to Figure 5). ${ }^{f}$ Too slow, too fast, or too small absorbance changes to be measured. ${ }^{g}$ M. Eder, F. Kräutle, P. Vock, V. Kieweg, J. Dong, I. Rasched, A. Strauss, J. J. P., Kim, and S. Ghisla, submitted.

to be reproducible when using different preparations of glu/ glu-MCADH and under variation of the conditions. It should also be noted that the specific activity of glu/glu-MCADH is close to that of MCADH with C8-CoA (Figure 2, righthand side panels). A major difference between the mutants (panel B) is the abrupt decrease of activity with glu/glu$\mathrm{MCADH}$ on increasing the chain length from $\mathrm{C} 10$ - to $\mathrm{C} 12$ CoA. This contrasts with MLCADH where $V_{\max }$ is maximal with the same substrates.

It should be noted that MCADH is the only enzyme of the acyl-CoA dehydrogenase family with a Thr at position 255 and concomitantly a Glu at position 376 . "Transposition" of Thr to position 376 and Glu to position 255 yielding 255Glu-376Thr-MCADH also results in a shift of the chain length activity profile to higher values; however, this also causes a drastic reduction of $V_{\max }$ (Table 1). While Thr255 probably has a specific role in MCADH, its identification is not possible from the present data. Mutants designed to address this specific point are presently under study.

Reaction of MLCADH and glu/glu-MCADH with Substrates of Varying Chain Length. MCADH's from different sources have been shown to bind substrates of varying chain length, upon which reduction of the oxidized flavin cofactor occurs concomitant with partial formation of the reduced enzyme-enoyl-CoA complex (Beinert \& Page, 1957; Thorpe et al., 1979) (Scheme 1). The latter is characterized by a long-wavelength charge transfer (CT) absorption extending up to $800 \mathrm{~nm}$ (Figure 3). As pointed out elsewhere (Schopfer et al., 1988) the extent of reduction of the flavin varies with substrate chain length. It reflects the internal equilibrium $K_{\mathrm{e}}$ of the couples of eq 1 and the affinities of $\mathrm{E}-\mathrm{Fl}_{\mathrm{ox}}$ and $\mathrm{E}-\mathrm{Fl}_{\mathrm{red}}$ for $\mathrm{S}$ and $\mathrm{P}\left(K_{\mathrm{S}}, K_{\mathrm{P}}\right.$, eq 1$)$, and for $\mathrm{P}$ and $\mathrm{S}\left(K_{\mathrm{P}^{\prime}}, K_{\mathrm{S}^{\prime}}\right.$, eq 2) respectively.

The ratio of oxidized/reduced flavin will be dependent on the combination of the equilibria of eqs 1 and 2; put into simpler terms an increasing affinity of $\mathrm{E}-\mathrm{Fl}_{\text {red }}$ for $\mathrm{P}$ (and $\mathrm{S}$ ) will shift the overall equilibrium to the right. Figure 3 illustrates the results of such experiments for MLCADH, and the percent reduction for the different ADH's using substrates of varying chain length is listed in Table 1 . The extent of reduction is a complex parameter, which reflects the thermodynamic constants included in eqs 1 and 2 (Schopfer et 


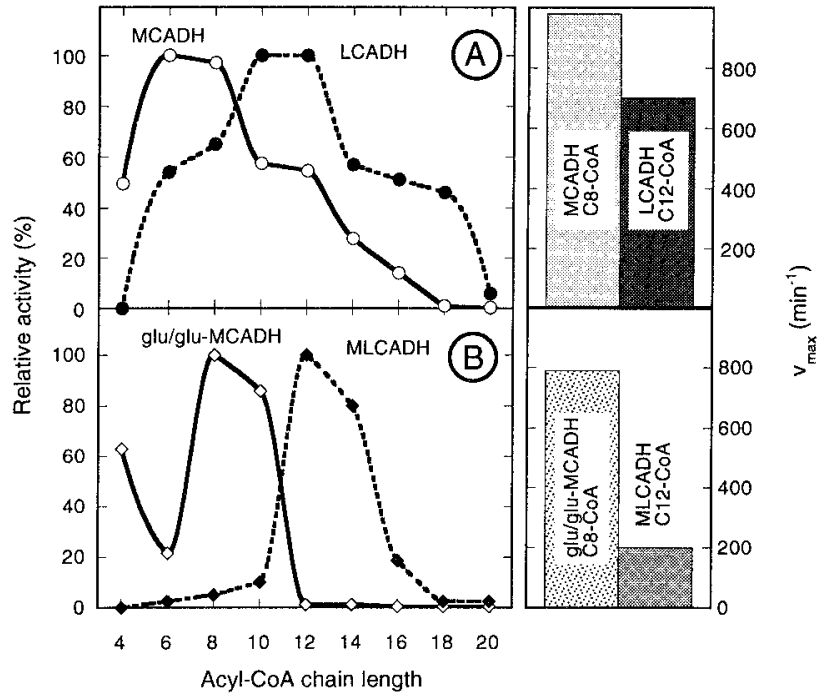

FIGURE 2: Chain length dependence of the activity of MLCADH and glu/glu-MCADH compared to that of MCADH and LCADH. The $V_{\max }$ values ( $c f$. Table 1) were normalized to $100 \%$ for the best substrate of each enzyme. (A) Chain length profiles of the activity of the wild type MCADH and LCADH and (B) profiles of MLCADH and glu/glu-MCADH. The $V_{\max }$ values for the best substrate of each enzyme are compared on the right hand site panels. The error for single data points is $\leq 10 \%$.

Scheme $1^{a}$

$$
\begin{aligned}
& \mathrm{E}-\mathrm{Fl}_{\mathrm{Ox}}+\mathrm{S} \stackrel{\mathrm{K}_{\mathrm{s}}}{\longleftrightarrow} \mathrm{E}-\mathrm{Fl}_{\mathrm{Ox}}-\mathrm{S} \stackrel{\mathrm{K}_{\mathrm{e}}}{\longleftrightarrow} \mathrm{E}-\mathrm{FI}_{\text {red }}-\mathrm{P} \stackrel{\mathrm{K}_{\mathrm{p}}}{\longleftrightarrow} \mathrm{E}-\mathrm{Fl}_{\text {red }}+\mathrm{P}(1) \\
& \mathrm{E}-\mathrm{Fl}_{\mathrm{ox}}+\mathrm{P} \stackrel{\mathrm{K}_{p^{\prime}}}{\longrightarrow} \mathrm{E}-\mathrm{Fl}_{\mathrm{ox}}-\mathrm{P} ; \mathrm{E}-\mathrm{FI}_{\text {red }}+\mathrm{S} \stackrel{\mathrm{K}_{s^{\prime}}}{\longrightarrow} \mathrm{E}-\mathrm{FI}_{\text {red }}-\mathrm{S} \\
& \Sigma \mathrm{E}-\mathrm{FI} \mathrm{red}_{\text {red }}+\mathrm{Acc}_{\mathrm{ox}} \stackrel{\mathrm{V}_{\text {reox }}}{\longrightarrow} \Sigma \mathrm{E}-\mathrm{FI}_{\mathrm{ox}}+\mathrm{Acc}_{\text {red }}
\end{aligned}
$$

${ }^{a} \mathrm{~S}=\mathrm{R}-\mathrm{CH}_{2}-\mathrm{CH}_{2}-\mathrm{COS}-\mathrm{CoA} ; \mathrm{P}=\mathrm{R}-\mathrm{CH}=\mathrm{CH}-\mathrm{COS}-\mathrm{CoA}$; $\mathrm{R}=$ alkyl chain of varying length; $\mathrm{ACC}=$ acceptor $\left[\mathrm{ETF}, \mathrm{O}_{2}\right.$, or Ferricenium]; $K=$ equilibrium constants for the steps and species shown; and $K_{\mathrm{e}}=k_{\mathrm{red}} / k_{\mathrm{ox}}, \sum \mathrm{E}=$ sum of all oxidized or reduced enzyme species. Note that eqs 1-3 are a simplification of the schemes described by Schopfer et al. (1988).

al., 1988). C18-CoA is an extreme example in that binding to form the Michaelis complex via $K_{\mathrm{S}}$ is demonstrated by the occurring spectral perturbation (Figure 3), while the extent of reduction is minimal. In spite of this, minimal activity $\left(V_{\max }<5 \mathrm{~min}^{-1}\right)$ with this substrate is observable (Table 1).

Reductive Half-Reaction. The chemically relevant step in the dehydrogenation of substrates by ADH's is the reductive one, which is initiated by $\mathrm{H}^{+}$-abstraction from the substrate $\mathrm{C}_{\alpha}$ by a Glu residue (Pohl et al., 1986; Powell \& Thorpe, 1988; Bross et al., 1990; Ghisla et al., 1984). The efficiency of this Glu as a base is thus likely to affect the rate of dehydrogenation $\left(k_{\text {red }}\right)$. The latter was determined for $\mathrm{MCADH}, \mathrm{MLCADH}$, and glu/glu-MCADH as a function of the substrate chain length using the stopped flow technique. The general behavior of the three proteins is similar, e.g., using C10-CoA as a substrate, the only one which could be measured under comparable conditions with all three proteins, dehydrogenation is accompanied by a biphasic OD-decrease at $450 \mathrm{~nm}$ (Figure 4). The first phase reflects reduction of the flavin cofactor/oxidation of the substrate in a concerted mechanism (Pohl et al., 1986; Schopfer et al., 1988). As shown in Table 1, the effect of transposing Glu from position 376 to 255 (MLCADH) reduces the rate of the first rapid phase 3-300-fold depending on the substrate chain length. With glu/glu-MCADH,

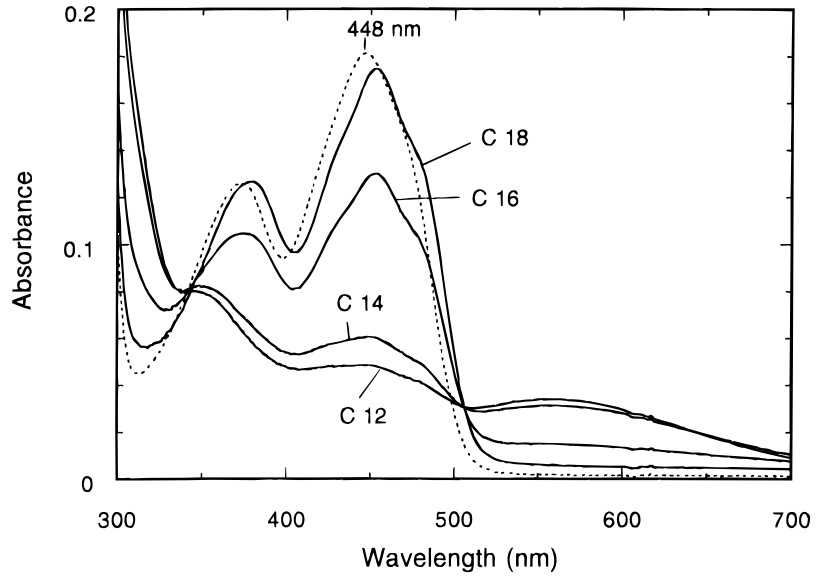

FIGURE 3: Reaction of MLCADH with substrates of varying chainlength and effect on the extent of reduction. To the oxidized enzyme, $13 \mu \mathrm{M}$ in standard buffer, (- - ) were added the acylCoA substrates with the indicated chain length $(130 \mu \mathrm{M}$ final concentrations), and the spectra were recorded after $\sim 2 \mathrm{~min}$, at which time spectral changes had ceased. The spectra obtained with C4-, C8-, and C10-CoA have intermediate character and for clarity are not shown ( $c f$. Table 1 for data). The extent of bleaching of the oxidized flavin band $\left(\lambda_{\max } \approx 450 \mathrm{~nm}\right.$ ) for the individual substrates is reported in Table 1 and is related to the bleaching obtained upon anaerobic reduction of oxidized MCADH with $\mathrm{C} 8-\mathrm{CoA}$ at the same wavelength, which is set to $100 \%$.

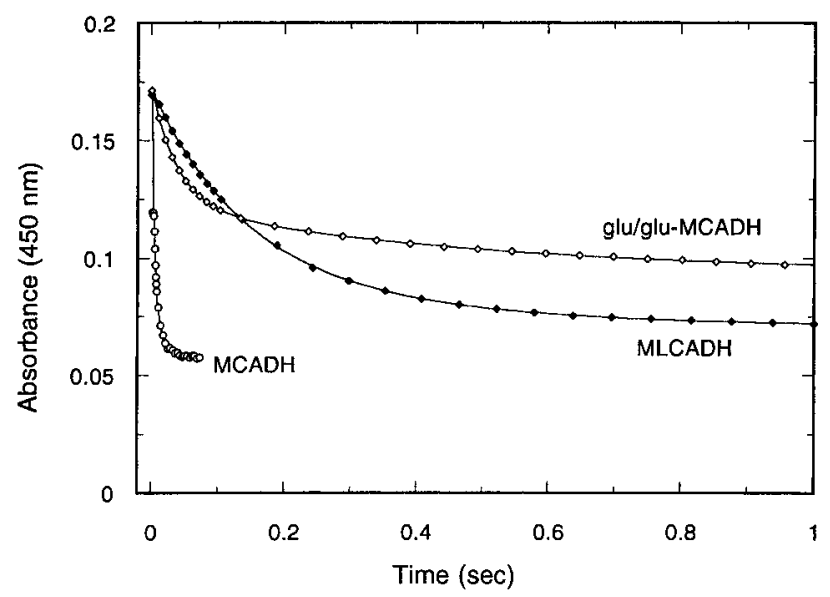

FIGURE 4: Time course of the reaction of MCADH (O), glu/gluMCADH $(\diamond)$, and MLCADH $(\diamond)$ with C10-CoA. The enzymes, $\sim 6 \mu \mathrm{M}$ in standard buffer and at $25^{\circ} \mathrm{C}$, were reacted with $100 \mu \mathrm{M}$ substrate (both final concentrations) in the stopped-flow instrument $\left(\mathrm{MCADH}\right.$ at $2{ }^{\circ} \mathrm{C}$ ). The data points shown are the numerical average from three to four single shots. The lines $(-)$ are the best fits obtained using a two exponential algorithm. Note that for MCADH the initial $\sim 50 \%$ of the reaction occur in the dead time of the instrument.

where Glu376 is assumed to be the working base ( $c f$. below), the value is intermediate and 1/10 that of MCADH for C10CoA. The values of $k_{\text {red }}$ compared to those of $V_{\max }$ (Table 1 and Figure 2) indicate that $k_{\text {red }}$ as such is not the sole factor responsible for overall catalysis as will be further elaborated in the discussion section.

Reaction of Reduced Enzymes with Oxygen. The reaction of the reduced enzyme/product complexes of pig MCADH with oxygen $\left(V_{\text {reox }}-\mathrm{O}_{2}\right)$ is extremely slow (Wang \& Thorpe, 1991) and in the order of hours depending on the substrate chain length. An important factor affecting $V_{\text {reox }-\mathrm{O}_{2}}$ is assumed to be the tightness of product binding, and in particular its rate of dissociation (Kumar \& Srivastava, 1994) which in turn can depend on the chemical constitution of 

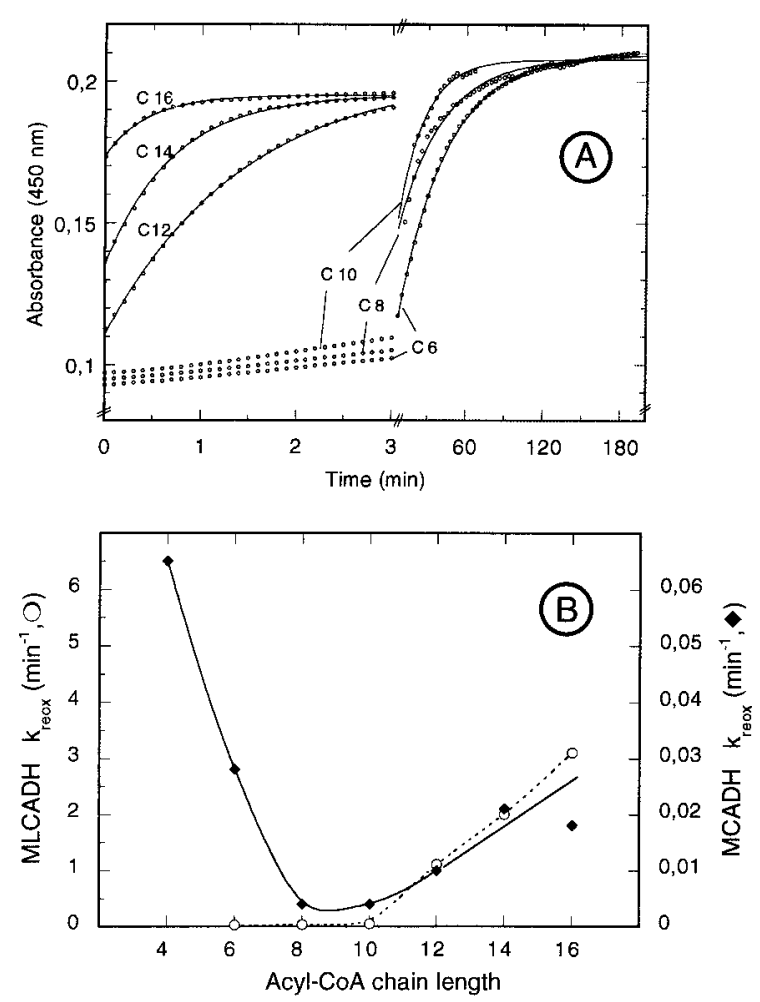

FIGURE 5: Course of reoxidation of MLCADH and MCADH following reduction with substrates of varying chain length. Panel A: Oxidized MLCADH, $\sim 14 \mu \mathrm{M}$ in standard buffer at $25^{\circ} \mathrm{C}$, was reacted aerobically with $\sim 0.7$ equiv of the acyl-CoA substrates with the indicated chain length. Note that the initial extent of reduction (time scale, ms; $c f$. Figure 4) depends on the substrate chain length (cf. Figure 3). The symbols are data points and the traces are the best fits obtained using monoexponential fit routines. Note that in particular with $\mathrm{C} 6, \mathrm{C} 8$, and $\mathrm{C} 10$ reoxidation occurs subsequent to a lag phase. Thereupon the increase of absorbance, which reflects (re)formation of oxidized enzyme, was recorded with time. The end-OD observed with C6-, C8-, and C10-CoA is somewhat higher due to formation of turbidity at $t>120 \mathrm{~min}$. Panel B shows the chain length dependence of the rate of "reoxidation", obtained from single-exponential fits of curves such as those in panel A, for $\operatorname{MLCADH}(\mathrm{O}$, left ordinate), and $\mathrm{MCADH}(\diamond$, right ordinate). Note the 100 -fold difference in the scales of both ordinates.

the substrate chain. We have estimated $V_{\text {reox }-\mathrm{O}_{2}}$ using enzyme reduced with substoichiometric amounts of substrate under the condition described in the legend of Figure 5, and in dependence of the substrate chain length. There are marked differences in $V_{\text {reox }-\mathrm{O}_{2}}$ of MLCADH compared to MCADH as evidenced by the plots of Figure 5B. First the reoxidation rate is 10-100-fold higher with MLCADH and C8- to C16-substrates. With MCADH there is an increase in $V_{\text {reox }-\mathrm{O}_{2}}$ using C6- and C4-CoA, which is not apparent with MLCADH (C6-CoA). On the other hand there is an abrupt increase in $V_{\text {reox }-\mathrm{O}_{2}}$ for both MCADH and MLCADH with substrates $>\mathrm{C} 10$. Thus with MCADH, but not with MLCADH, the chain length dependence of $V_{\text {reox }-\mathrm{O}_{2}}$ has the $\mathrm{U}$-shaped profile shown in Figure 5B. It is worth noting that as a first approximation this profile of $V_{\text {reox }-\mathrm{O}_{2}}$ has the opposite dependence of that of $V_{\max }$ shown in Figure 2. These results can be rationalized by assuming an effect of the chain length on $K_{\mathrm{S}}, K_{\mathrm{P}}, K_{\mathrm{S}^{\prime}}$ and $K_{\mathrm{P}^{\prime}}$ (eqs 1 and 2) and on the underlying rates of association/dissociation with a specific "break" at a chain length of $\sim \mathrm{C} 10$ (see Discussion). These factors will affect the "availability" of the reduced enzyme species $\mathrm{E}-\mathrm{Fl}_{\mathrm{red}}-\mathrm{P}, \mathrm{E}-\mathrm{Fl}_{\mathrm{red}}$ as well as of further, connected intermediates such as those postulated by Schopfer et al.

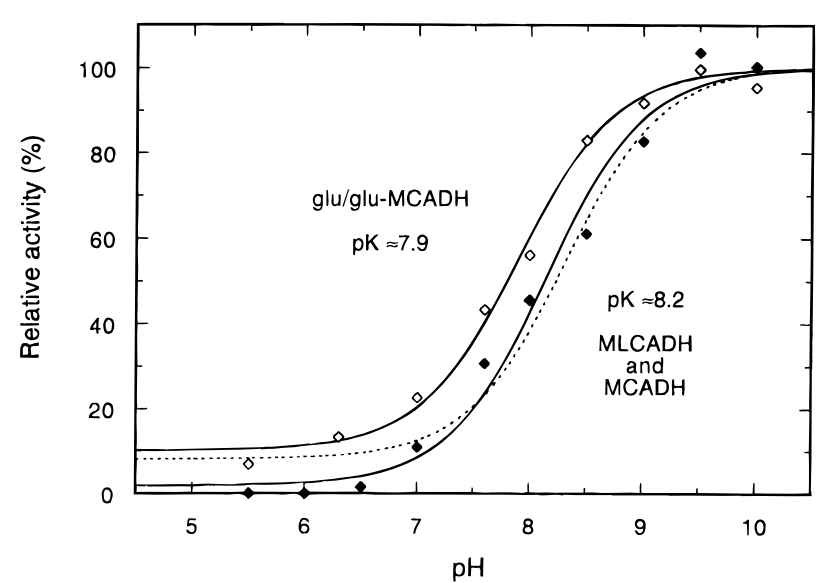

FIGURE 6: $\mathrm{pH}$ Dependence of the activity of glu/glu-MCADH and MLCADH. The activity (ferricenium assay) was tested at fixed and saturating concentrations of $100 \mu \mathrm{M} \mathrm{C} 8-\mathrm{CoA}$ (glu/glu-MCADH) and C12-CoA (MLCADH) in $100 \mathrm{mM}$ MES, MOPS, TAPS, and CHES buffers adjusted at $\mathrm{pH}$ values shown (the activity was found not to depend on the ionic strength $(\mathrm{KCl})$ of the medium in the $0.01-0.1 \mathrm{M}$ range). The observed velocities are standardized to $100 \%$ for comparison. The extrapolated maximal $/$ minimal velocities were $1140 / 155$ and $660 / 12\left[\mathrm{~min}^{-1}\right.$ ] for glu/glu-MCADH $(\diamond)$ and MLCADH $(\diamond)$, respectively, and were obtained from the $\mathrm{pH}$ activity fits represented by the curves and using the standard velocity $-\mathrm{pH}$ dependence equation. The curve for MCADH (- - -) is shown for comparison and was taken from Ghisla et al. (1994).

(1988) and Kumar and Srivastava (1994), which are all species potentially reactive with ferricenium and oxygen as electron acceptors. There is no dependence of $V_{\text {reox }-\mathrm{O}_{2}}$ on the $\mathrm{O}_{2}$ concentration (not shown), in accordance with the assumption that dissociation of product, or the concentration of a species directly linked to it, is limiting in $\mathrm{O}_{2}$ reoxidation.

pH dependence of the Activity. Glu376 initiates catalysis by $\mathrm{H}^{+}$-abstraction from substrates in MCADH (Powell \& Thorpe, 1988; Bross et al., 1990). Its $\mathrm{p} K$ and the factors which modulate it are thus of particular interest. The $\mathrm{pH}$ dependence of the activity reflects a $\mathrm{p} K 7.5-8.5$, which can be attributed to the ionization of Glu376 (Ghisla et al., 1994), implying an increase by $\sim 4$ units compared to that of free Glu-COOH ( $\mathrm{p} K \approx 4.3$ ). For MLCADH the same $\mathrm{p} K \approx 8.2$ can be estimated from similar experiments (Figure 6). This indicates that the position of the Glu does not affect this $\mathrm{p} K$, and that the $376-$ and $255 \mathrm{Glu}-\mathrm{COO}^{-}$-base is located very much in the same microenvironment during catalysis (Figure 1). With glu/glu-MCADH the same $\mathrm{p} K$ is lowered to $\sim 7.9$, which can reasonably be attributed to an increased local polarity induced by the presence of the two Glu's.

Is MLCADH More Related to MCADH or to LCADH? Reactions with Substrate Analogs. Some of the differences in chemical reactivity between MCADH and LCADH could originate from the different location of Glu376 and Glu255 with respect to the substrate and the flavin. 2-OctynoylCoA inactivates pig kidney MCADH via formation of a covalent adduct with Glu376. This is accompanied by appearance of a long-wavelength CT-band, which subsequently decays slowly with simultaneous elimination of CoASH (Powell \& Thorpe, 1988). Human LCADH is inactivated by 2-octynoyl-CoA, but without appearance of a CT-species (unpublished data). This contrasts with earlier experiments done with beef liver LCADH, from which it was deduced that this LCADH is apparently not inactivated by 2-octynoyl-CoA (Ankele et al., 1991). The experiments depicted in Figure 7A show that the course of the reaction 

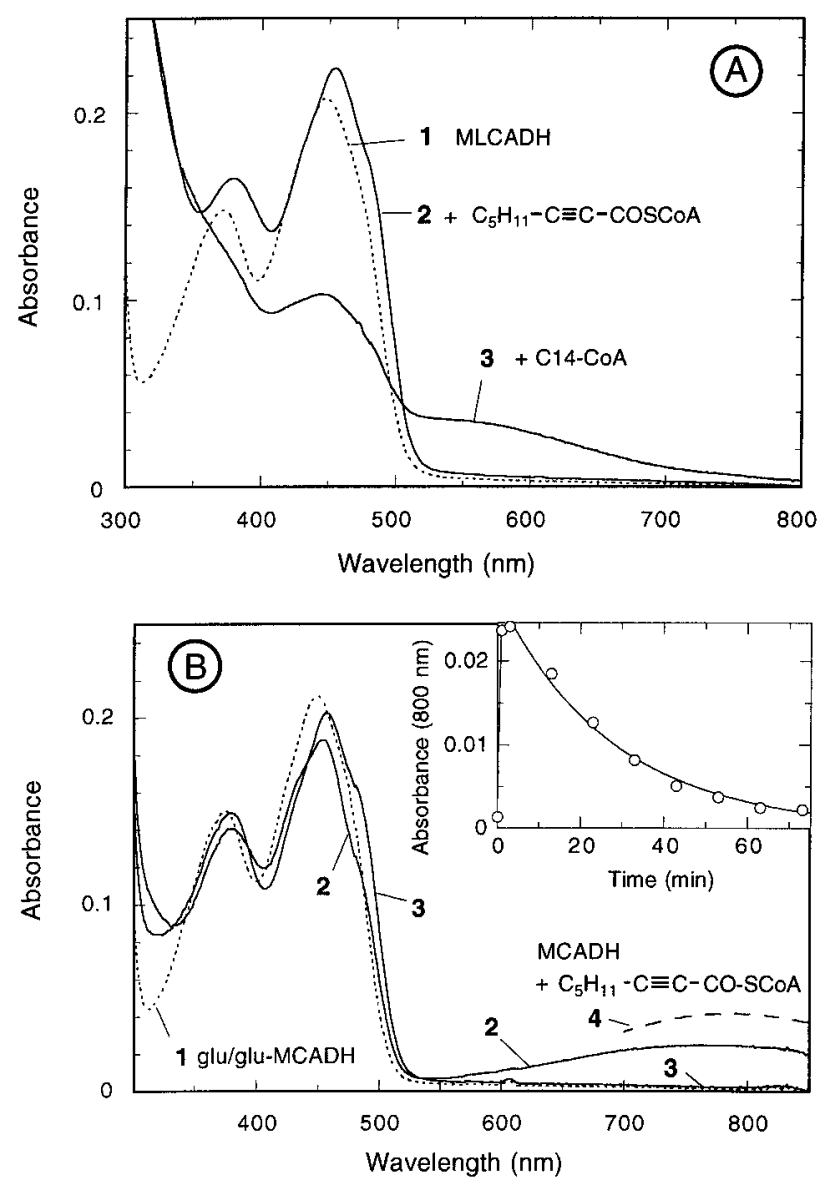

FIGURE 7: (A) Reaction of MLCADH with 2-octynoyl-CoA and subsequently with C14-CoA. Oxidized enzyme, $15 \mu \mathrm{M}$ in standard buffer, curve 1 (- - ), was reacted with $250 \mu \mathrm{M}$ 2-octynoyl-CoA and the spectrum of curve 2 was recorded upon 2 min. Subsequent addition of $100 \mu \mathrm{M} \mathrm{C14-CoA}$ yields the spectrum of curve 3 , which is that of (partially) reduced MLCADH (compare to Figure 3). (B) Reaction of glu/glu-MCADH with 2-octynoyl-CoA. Spectral changes observed on the addition of 2-octynoyl-CoA to glu/glu-MCADH are shown. The oxidized dehydrogenase (1) in $50 \mathrm{mM}$ phosphate buffer, $\mathrm{pH} 7.6(15 \mu \mathrm{M})$ was treated with $20 \mu \mathrm{M}$ 2-octinoyl-CoA; maximal formation of the $800 \mathrm{~nm}$ band (2) 3 min after mixing; final spectrum after $73 \mathrm{~min}$ (3). The inset shows the absorbance changes at $800 \mathrm{~nm}$. For comparison the maximal formation of the charge transfer band $(800 \mathrm{~nm})$ with $\mathrm{MCADH}$ is shown (4). Addition of $100 \mu \mathrm{M}$ C8-CoA shows no reduction.

of MLCADH with 2-octynoyl-CoA is markedly different from that of MCADH but more similar to that of human LCADH. No intermediate species with CT-absorption is observed, and no covalent inactivation occurs since, upon subsequent addition of C14-CoA, flavin reduction ensues readily (Figure 7A). This behavior also contrasts with that observed using glu/glu-MCADH and as shown in Figure 7B. Here, and in analogy to MCADH, a long-wavelength band is formed rapidly, which subsequently decays monophasically and at a much slower rate $\left(t_{1 / 2} \approx 20 \mathrm{~min}\right)$. Subsequent addition of excess C8-CoA has no effect on the spectrum of the oxidized flavin. The overall behavior and the decay rate are similar to what has been reported by Powell and Thorpe (1988) for pig MCADH $\left(t_{1 / 2} \approx 13 \mathrm{~min}\right)$. These results are thus consistent with Glu376 being the one involved in catalysis in the case of glu/glu-MCADH, as opposed to Glu255, and with the occurrence of the same mechanism of inactivation as with MCADH, i.e., covalent modification of Glu376 by the inhibitor (Powell \& Thorpe, 1988).

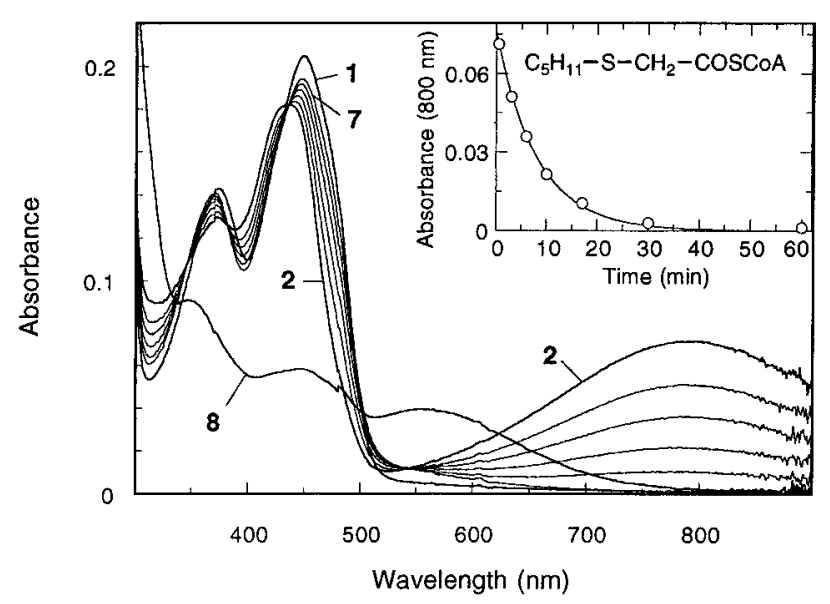

Figure 8: Reaction of MLCADH with 3-thiaoctanoyl-CoA: consumption without loss of activity. The oxidized enzyme, $15 \mu \mathrm{M}$ in standard buffer, curve 1 , was reacted with $16 \mu \mathrm{M} 3$-thiaoctanoyl$\mathrm{CoA}$. The spectrum obtained within the mixing time is that of curve 2 , which is characterized by the high (CT) absorbance centered at $\sim 785 \mathrm{~nm}$. This species decays isosbestically and monophasically (insert) with a $t_{1 / 2} \approx 6 \mathrm{~min}$ to yield the species represented by curve 7. This process can be repeated by successive additions of 3-thiaoctanoyl-CoA (not shown). Final addition of $100 \mu \mathrm{M}$ octanoyl-CoA yields curve 8, which is that of reduced MLCADH complexed with octenoyl-CoA (compare to Figure 2).

3-Thiaoctanoyl-CoA binds to MCADH $\left(K_{\mathrm{d}} \approx 0.5 \mu \mathrm{M}\right)$ and then undergoes deprotonation at position $\alpha$ forming a stable CT-complex with a $\lambda_{\max } \approx 785 \mathrm{~nm}$ (Lau et al., 1988). MLCADH also binds the analog $\left(K_{\mathrm{d}} \approx 0.3 \mu \mathrm{M}\right.$ determined as detailed in the legend of Figure 8 ) and the CT complex is formed within seconds. However, in contrast to the case of MCADH, the complex is not stable and decays monophasically with a half-time of $\sim 6 \mathrm{~min}$ at $25^{\circ} \mathrm{C}$ (Figure 8 , inset). This sequence can be repeated several times indicating that it does not involve modification of the enzyme. In accordance with this, final addition of $\mathrm{C} 8$-CoA leads to immediate reduction of the enzyme flavin (Figure 8, curve 8). Although the exact nature of the products of conversion of 3-thiaoctanoyl-CoA is not known, we assume that the decay process involves elimination of CoASH as observed with some similar substrate analogs (Lau et al., 1988).

4-Nitrophenylacetyl-CoA is a related analog with which $\alpha-\mathrm{H}$ abstraction can be observed directly and which has been employed for estimation of the microscopic $\mathrm{p} K$ shifts induced by binding to the active center of MCADH (Engst, 1993). Upon binding to MLCADH, the analog is converted to the anionic form $\left(\lambda_{\max } \approx 507 \mathrm{~nm}\right)$ which induces a CT-band centered at $\sim 750 \mathrm{~nm}$ (Figure 9). This is similar to what is observed with MCADH (Engst \& Ghisla, 1991) and occurs with all mutants (not shown). However, binding to ML$\mathrm{CADH}$ is approximately ten times tighter than to MCADH and comparable to binding to E376Q-MCADH (Bross et al., 1990; Engst, 1993) ( $K_{\mathrm{d}}: \mathrm{MCADH}, \sim 23 \mu \mathrm{M}$; MLCADH $\sim 2$ $\mu \mathrm{M}$, glu/glu-MCADH, $\sim 26 \mu \mathrm{M}$; and E376Q-MCADH, $\sim 2$ $\mu \mathrm{M})$. It thus appears that the charge of Glu376 affects binding of this analog.

\section{DISCUSSION}

The principal point emerging from the present study is that transposition of the catalytic base Glu on loop JK (position 376) to helix G (position 255, Figure 9) leads to mutants with catalytic activity comparable to that of the 


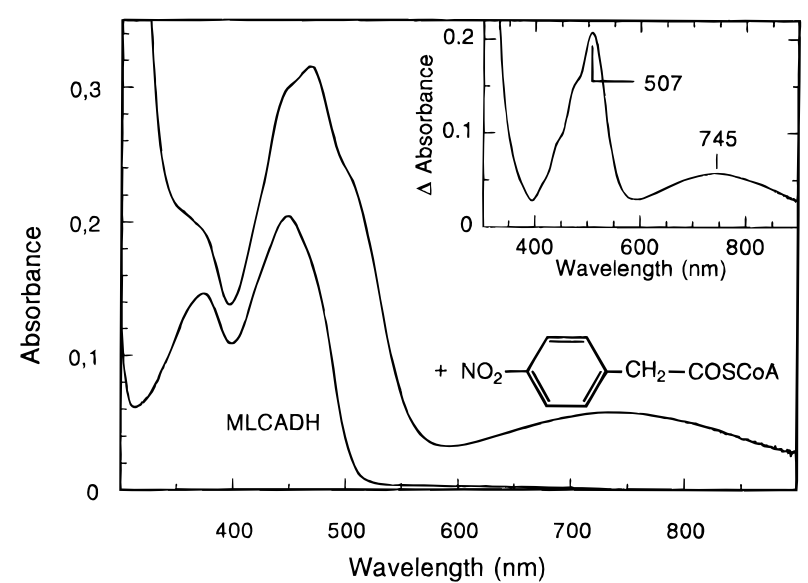

FIGURE 9: Interaction of MLCADH with 4-nitrophenylacetyl-CoA. To the oxidized enzyme, $15 \mu \mathrm{M}$ in standard buffer, incremental amounts of a solution of 4-nitrophenyl-acetyl-CoA in the same buffer were added. The upper curve represents the spectrum (corrected for dilution) obtained in the presence of $133 \mu \mathrm{M}$ of the reagent (final concentration), which corresponds to saturating conditions. The inset shows the difference spectrum between uncomplexed and complexed MLCADH. Note that the band centered around $507 \mathrm{~nm}$ is attributed to the ligand anion, and that around $745 \mathrm{~nm}$ to the CT complex between the latter and the oxidized enzyme flavin.

parent MCADH (Figure 2, Table 1). For an assessment of the effects of mutations on catalysis and substrate specificity, it is of advantage to analyze the kinetic constituents of $V_{\max }$ and in particular the steps involved in the reductive and in the oxidative half reactions individually (Beinert, 1963), (Thorpe et al., 1979). While the rate-limiting components of the reductive half-reaction, $k_{\text {red }}$, are quite well defined (Schopfer et al., 1988; Kumar \& Srivastava, 1994; Pohl et al., 1986), those of the oxidative half-reaction are not and have not been measured directly in the present work nor in previous ones. Thus, with "good substrates", the rate of $k_{\text {red }}$ appears to be much higher compared to $V_{\max }$, and the reoxidation half-reaction $\left(V_{\text {reox }}\right.$, Scheme 1$)$ is rate limiting (Table 1). For MCADH a reduction rate $k_{\text {red }}$ of $24000 \mathrm{~min}^{-1}$ at $2{ }^{\circ} \mathrm{C}$ was determined while $V_{\max }$, using the ferricenium assay, is only $\sim 1000 \mathrm{~min}^{-1}$. Conversely with "poor substrates" and when $V_{\max }<500 \mathrm{~min}^{-1}, k_{\text {red }}$ approaches limiting rates. A case in point is MLCADH with C14-CoA, where $V_{\max }$ was estimated as $\sim 160 \mathrm{~min}^{-1}$ and $k_{\text {red }}$ as $\sim 150$ $\min ^{-1}$. The differences in the observed activities are thus affected by the variation/combination of $k_{\text {red }}$ and $V_{\text {reox. }}$. In addition, these two factors are affected by the concentrations of the intermediary species via the equilibria of eqs 1 and 2 (Scheme 1).

For glu/glu-MCADH and with C8-substrate, the rate of enzyme flavin reduction, $k_{\text {red }}$, is of the same order as for $\mathrm{MCADH}$; with $\mathrm{C} 10-\mathrm{CoA}$, it decreases $\geq 10$-fold and with $>$ C10-substrates it is close to zero (Table 1). Remarkably, and in agreement with $V_{\text {reox }}$ (acceptor: ferricenium) being rate limiting, $V_{\max }$ is essentially the same for $\mathrm{C} 8$ - and $\mathrm{C} 10$ substrates (Figure 2), while $k_{\text {red }}$ differs by 1 order of magnitude (Table 1). Also, glu/glu-MCADH differs little from MCADH with respect to $V_{\max }$ and $V_{\text {reox }} \mathrm{O}_{2}$ (Table 1). This might result from a steric or charge effect induced by the "additional" Glu255-side chain in glu/glu-MCADH since the capacity of $376 \mathrm{Glu}-\mathrm{COO}^{-}$to abstract the substrate $\alpha-\mathrm{H}$ (or the subsequent $\beta$-hydride transfer to the flavin) appears to be impaired only when the substrate chain length is $>\mathrm{C} 10$.
The increase of $K_{\mathrm{m}}$ with increasing chain length might reflect an impairment of catalytically important interactions with substrates $>$ C10. In contrast, the active site of MLCADH, (with the configuration Glu255-Gly376), has no difficulties in accommodating such substrates. It also binds the substrate analog, 4-nitrophenyl-acetyl-CoA, $\sim 10$-fold better than $\mathrm{MCADH}$, because the analog should have steric requirements more extensive than those of C8-CoA (Figure 9). This interpretation is supported by the $3 \mathrm{D}$ data presented in the accompanying paper (Lee et al., 1996).

In contrast to these results, $k_{\text {red }}$ values of MLCADH are decreased by approximately 2 orders of magnitude compared to those of MCADH, but they vary only $\sim 3$ fold for C8-, C10-, C12-, and C14-CoA and are maximal with C10- and $\mathrm{C} 12$-substrates. Conversely $V_{\text {reox }-\mathrm{O}_{2}}$ is $\approx 100$-fold faster with MLCADH compared to MCADH (Table 1, Figure 5B). The chain length dependence of $V_{\text {reox }}-\mathrm{O}_{2}$ is quite peculiar; with both enzymes there is a sharp increase of $V_{\text {reox }}-\mathrm{O}_{2}$ between $\mathrm{C} 10$ - and $\mathrm{C} 12$-substrates, suggesting the occurrence of a chain length dependent "cutoff" limit. It has been shown that, in the absence of substrate at the active site of MCADH, a string of five water molecules occupies the place of the substrate alkyl chain (Kim et al., 1994). Importantly, the number of $\mathrm{H}_{2} \mathrm{O}$ molecules displaced by substrate depends linearly from the length of its chain (Kim et al., 1994). The observed "break" in $V_{\text {reox }-\mathrm{O}_{2}}$ (Figure 5B) is thus likely to correspond to the length of the chain at which all water molecules are displaced, i.e., $\sim \mathrm{C} 10$. With substrate chains exceeding $\mathrm{C} 10$, the protein probably must undergo conformational adjustments in order to accommodate the increased size of the alkane chain. As a consequence, affinity for ligands with $>\mathrm{C} 10$ does decrease, this resulting from an increase of the rate of dissociation. Our results should be compared with an earlier report by Wang and Thorpe (1991), who state that "in general lengthening of the chain increases the protection." However, these authors measured reoxidation rates in the presence of excess thioether ligands, while in the present case the rate of reoxidation is probably affected by the interaction of product $\beta$-ketoacid (formed from dehydrogenation of substrate) with reduced enyzme. Our results concur with those of Trievel et al. (1995), in which the chain length dependence on the free energy of binding shows a "break" at C8-C10 for various analogs binding to oxidized pig MCADH.

With MCADH, and in the context of chain length dependence, $V_{\text {reox }-\mathrm{O}_{2}}$ is minimal when $V_{\max }$ is maximal (compare Figures 2 and 5B). A striking observation is the overall $\sim 100$-fold increase of $V_{\text {reox }-\mathrm{O}_{2}}$ with MLCADH compared to MCADH (Figure 5B). This probably does originate from an increased rate of ligand dissociation from reduced enzyme with MLCADH due to specific steric interferences. The interpretation that factors affecting the rates of dissociation of product influence $V_{\text {reox }-\mathrm{O}_{2}}$ is in line with the proposal of Johnson et al. (1994) that $V_{\text {reox }-\mathrm{O}_{2}}$ is limited by the rate of product dissociation. It is more difficult to be reconciled with the proposal by Thorpe's group (Wang $\&$ Thorpe, 1991) according to which stabilization of the incipient $\mathrm{O}_{2}{ }^{-}$is the crucial factor governing $\mathrm{O}_{2}$ reactivity in acyl-CoA dehydrogenases. On the other hand it is well established that the reactivity of reduced flavin with dioxygen is strongly affected by the properties of solvent (Müller et al., 1975). Thus, product dissociation rates might regulate 
the accessibility of solute to the active center and in turn affect $\mathrm{O}_{2}$ reactivity.

This interpretaion contrasts with that which invokes factors related to solvent polarity as affecting the rate of $V_{\text {reox }}-\mathrm{O}_{2}$. The identity of the pK's of Glu376 and Glu255 (Figure 6) suggests that the polarity at the active center is essentially unaltered, however, the difference in $V_{\text {reox }}-\mathrm{O}_{2}$ is $>100$-fold. An additional Glu, as in glu/glu-MCADH, should increase the polarity of the active center as reflected by the decrease of the apparent $\mathrm{p} K$ on $V_{\max }$ (Figure 6). This change, however, does have little effect on $V_{\text {reox }-\mathrm{O}_{2}}$. Unfortunately, the parameters with substrates $>\mathrm{C} 10$ cannot be measured with glu/glu-MCADH due to lack of activity (Table 1).

An important difference between MCADH and the mutants is the extent of reduction which occurs upon addition of excess substrate (Figure 3 ) and is assumed to reflect the equilibria of eqs 1 and 2 in Scheme 1. With MCADH and its best substrates, flavin reduction is essentially complete and fairly constant. However, it is less extensive with MLCADH, in particular, at the extremes of chain length $(<\mathrm{C} 8-,>\mathrm{C} 14-\mathrm{CoA}$, Table 1). Such data for LCADH are not yet available. These differences are assumed to reflect chain length variations in the substrate/product binding equilibria to $E_{\mathrm{ox}} / E_{\text {red }}$ as shown in Scheme 1.

It thus appears that the presence of a large functional group in addition to Glu376 renders the steric constraints at the active site of MCADH more stringent. Consequently, with MLCADH (Glu255, Gly376) both activity and $k_{\text {red }}$ have a broader spectrum than with glu/glu-MCADH. For 255Glu/ 376Thr-MCADH it is likely that the Thr376 side chain adversely affects positioning of the $\mathrm{Glu} 255-\mathrm{COO}^{-}$for efficient $\mathrm{H}^{+}$-abstraction. As detailed in the accompanying paper by Lee et al. (1996), in the crystals of MLCADH, two active center populations coexist in which Glu255 is either in contact with the substrate or "swings away" from the position required for catalysis (Kim et al., 1993). With glu/ glu-MCADH, Glu255-COOH might occupy an analogous "inactive" position and exist in its neutral form as can be deduced from the minor effect observed on the $\mathrm{pH}$ dependence of $V_{\max }$ (Figure 6). In turn, this requires that its $\mathrm{p} K$ be increased by at least a similar extent as that of Glu376.

The high activity of glu/glu-MCADH make the Glu255Glu376 mutation a likely candidate for a progenitor of LCADH and IVDH. LCADH and IVDH with the catalytic Glu on helix G form a single branch in the ADH/AOX superfamily, while in the ancestral gene from which the superfamily originated Glu was on the JK loop. A phylogenetic tree accounting for these hypotheses has been presented elsewhere (Nandy et al., 1996). On the other hand the position of Glu, either on loop JK or on helix G, is not a prerequisite for development of chain length specificity since the Glu in question is also on loop JK in very long chain acyl-CoA dehydrogenase (Aoyama et al., 1994).

Finally, the $\mathrm{H}^{+}$-abstraction catalyzed by acyl-CoA dehydrogenases appears to require a peculiar type of base machinery compared to other enzymes, where pairs or triads of functional groups are common. Glu376 (or Glu262 in LCADH) is not in close contact with any functional group capable of the above mentioned ancillary functions. The closest charged residue is Arg256 at a distance $>4.5 \AA$, and thus not at a reasonable H-bonding distance (Lee et al., 1996). The present results and the 3D structure of MLCADH (Lee et al., 1996) confirm the absence of such secondary interac- tions. Conversely, this absence might be one factor that allows for different topologies of the Glu base in question. To our knowledge, only in the lipase family, has a positional difference of a catalytically essential residue been reported (Schrag et al., 1992).

\section{ACKNOWLEDGMENT}

We thank Michael Eder for providing some of the data on hwtLCADH.

\section{REFERENCES}

Amann, E., Ochs, B., \& Abel, K. J. (1988) Gene 69, 301-315.

Ankele, K., Melde, K., Engst, S., Bross, P., Ghisla, S., \& Strauss, A. W. (1991) in Flavins and Flavoproteins, Proceedings of the Tenth International Symposium, Como, Italy (Curti, B., Ronchi, S., \& Zanetti, G., Eds.) pp 325-328, Walter de Gruyter \& Co., Berlin.

Aoyama, T., Ueno, I., Kamijo, T., \& Hashimoto, T. (1994) J. Biol. Chem. 269, 19088-19094.

Beinert, H. (1963) in The Enzymes (Boyer, P. D., Lardy, H., \& Myrback, K., Eds.) pp 447-476, Academic Press, New York. Beinert, H., \& Page, E. (1957) J. Biol. Chem. 225, 479-497.

Bisswanger, H. (1994) in Enzymkinetik, pp 22-26, VCH Verlagsgesellschaft, Weinheim.

Bross, P., Engst, S., Strauss, A. W., Kelly, D. P., Rasched, I., \& Ghisla, S. (1990) J. Biol. Chem. 265, 7116-7119.

Decker, K. (1959) Die aktivierte Essigsäure (Pummerer, R., Ed.) F. Enke, Stuttgart.

Djordjevic, S., Dong, Y., Paschke, R., Frerman, F. E., Strauss, A. W., \& Kim, J. J. P. (1994) Biochemistry 33, 4258-4264.

Engst, S. (1993) Thesis, University of Konstanz, Konstanz, Germany.

Engst, S., \& Ghisla, S. (1991) in Flavins and Flavoproteins, Proceedings of the Tenth International Symposium, Como, Italy (Curti, B., Ronchi, S., \& Zanetti, G., Eds.) pp 311-314, Walter de Gruyter \& Co., Berlin.

Fendrich, G., \& Abeles, R. H. (1982) Biochemistry 21, 6685-6695.

Ghisla, S., Thorpe, C., \& Massey, V. (1984) Biochemistry 23, 3154-3161.

Ghisla, S., Engst, S., Vock, P., Kieweg, V., Bross, P., Nandy, A., Rasched, I., \& Strauss, A. W. (1994) in Flavins and Flavoproteins, Proceedings of the Eleventh International Symposium, Nagoya, Japan (Yagi, K., Ed.) pp 283-292, Walter de Gruyter $\&$ Co., Berlin.

Gregersen, N., Winter, V., Curtis, D., Deufel, T., Mack, M., Hendrickx, J., Willems, P. J., Ponzone, A., Parrella, T., Ponzone, R., Ding, J. H., Zhang, W., Chen, Y. T., Kahler, S., Roe, C. R., Kølvraa, S., Schneiderman, K., Andresen, B. S., Bross, P., \& Bolund, L. (1993) Hum. Hered. 43, 342-350.

Johnson, J. K., Kumar, N. R., \& Srivastava, D. K. (1994) Biochemistry 33, 4738-4744.

Kim, J. J. P., \& Wu, J. (1988) Proc. Natl. Acad. Sci. U.S.A., 85, 6677-6681.

Kim, J. J. P., Wang, M., \& Paschke, R. (1993) Proc. Natl. Acad. Sci. U.S.A. 90, 7523-7527.

Kim, J. J. P., Wang, M., Djordjevic, S., Paschke, R., \& Bennett, D. W. (1994) in Flavins and Flavoproteins, Proceedings of the Eleventh International Symposium, Nagoya, Japan (Yagi, K., Ed.) pp 273-282, Walter de Gruyter \& Co., Berlin.

Kumar, N. R., \& Srivastava, D. K. (1994) Biochemistry 33, 88338841.

Langkau, B. (1993) Thesis, University of Konstanz, Konstanz, Germany.

Lau, S. M., Brantley, R. K., \& Thorpe, C. (1988) Biochemistry 27, 5089-5095.

Lee, H. K., Wang, M., Paschke, R., Nandy, A., Ghisla, S., \& Kim, J. J. P. (1996) Biochemistry 35, 12412-12420.

Lehman, T. C., \& Thorpe, C. (1990) Biochemistry 29, 1059410602.

Mohsen, A.-W., \& Vockley, J. (1995) Biochemistry 34, 1014610512 . 
Müller, F., Jarbandhan, T., Gast, R., \& Grande, H. J. (1975) in Reactivity of Flavins (Yagi, K., Ed.) pp 51-70, University of Tokyo Press, Tokyo.

Nandy, A., Bross, P., Kräutle, F.-G., Rasched, I., \& Ghisla, S. (1994) in Flavins and Flavoproteins, Proceedings of the Eleventh International Symposium, Nagoya, Japan (Yagi, K., Ed.) pp 327330, Walter de Gruyter \& Co., Berlin.

Nandy, A., Küchler, B., \& Ghisla, S., (1996) Biochem. Soc. Trans. 24, 105-110.

Pohl, B., Raichle, T., \& Ghisla, S. (1986) Eur. J. Biochem. 160, 109-115.

Powell, P. J., \& Thorpe, C. (1988) Biochemistry 27, 8022-8028.

Raichle, Th. (1981) Thesis, University of Konstanz, Konstanz, Germany.
Schopfer, L. M., Massey, V., Ghisla, S., \& Thorpe, C. (1988) Biochemistry 27, 6599-6611.

Schrag, J. D., Winkler, F. K., \& Cygler, M. (1992) J. Biol. Chem. 267, 4300-4303.

Thorpe, C., Matthews, R. G., \& Williams, C. H. J. (1979) Biochemistry 18, 331-337.

Trievel, R. C., Wang, R., Anderson, V. E., \& Thorpe, C. (1995) Biochemistry 34, 8597-8605.

Wang, R., \& Thorpe, C. (1991) Biochemistry 30, 7895-7901.

Whitby, L. G. (1953) Biochem. J. 54, 437-442.

Williamson, G., Engel, P. C., Mizzer, J. P., Thorpe, C., \& Massey, V. (1982) J. Biol. Chem. 257, 4314-4320. 